\title{
Asymptotic Statistical Properties of AR Spectral Estimators for Processes With Mixed Spectra
}

\author{
Soon-Sen Lau, Peter J. Sherman, Member, IEEE, and Langford B. White, Senior Member, IEEE
}

\begin{abstract}
In this paper, the influence of a point spectrum on large sample statistics of the autoregressive (AR) spectral estimator is addressed. In particular, the asymptotic distributions of the AR coefficients, the innovations variance, and the spectral density estimator of a finite-order $\operatorname{AR}(p)$ model to a mixed spectrum process are presented. Various asymptotic results regarding AR modeling of a regular process with a continuous spectrum are arrived at as special cases of the results for the mixed spectrum setting. Finally, numerical simulations are performed to verify the analytical results.
\end{abstract}

Index Terms-Asymptotic normality, autoregressive (AR) model, mixed spectrum processes, spectral analysis.

\section{INTRODUCTION}

A UTOREGRESSIVE (AR) models have been well studied in relation to regular stochastic processes containing an absolutely continuous spectrum. For example, [1] derived the asymptotic normality of the coefficients of an $\operatorname{AR}(p)$ model for an $\operatorname{AR}(q)$ process with $p \geq q$ by using linear regression, while [2] employed the convergence properties of martingale differences to arrive at the limiting distribution of the parameters of a $p$ th-order predictor for a regular stationary process. In frequency-domain analysis, AR models have received extensive interest as high-resolution alternatives to spectral estimates based on the Fourier transform of the data. In [3] and [4], confidence intervals were established for an AR spectral estimator in the case of a regular process. The limiting distribution of the AR spectral density estimator for a regular causal stationary process when both $p$ and the sample size approach infinity under certain conditions was approximated in [5].

Mixed spectrum processes, which contain both an absolutely continuous spectrum and point masses associated with sinusoids, exist in a wide variety of practical situations such as communications, radar, rotating machinery, meteorology, climatology, and geoscience. In spite of this, the limiting statistical properties of AR spectral analysis for mixed spectrum processes have received limited attention. In [6], the asymptotic

Manuscript received April 7, 2000; revised November 26, 2001. This work was supported under Grant AFOSR-F496209510090.

S.-S. Lau is with Qualcomm, Inc., San Diego, CA USA (e-mail: slau@qualcomm.com)

P. J. Sherman is with the Department of Aerospace Engineering and Engineering Mechanics, Iowa State University, Ames, IA 50010 USA (e-mail:shermanp@iastate.edu).

L. B. White is with the Defense Science and Technology Organization, Australia and Adelaide University, Adelaide, Australia (e-mail: lang.white@adelaide.edu.au).

Communicated by J. A. O'Sullivan, Associate Editor for Detection and Estimation.

Publisher Item Identifier S 0018-9448(02)01938-7. variance of the fixed $p$ th-order AR spectral estimator for a general mixed spectrum process was developed under the assumption of small estimation error. For a single sinusoid plus white noise, [7] proved that for fixed $p$, the least squares $\operatorname{AR}(p)$ spectral estimator converges almost surely to the spectrum of the theoretical $p$ th-order predictor. They also proved that the theoretical $\operatorname{AR}(p)$ spectrum becomes unbounded at a signal frequency as $p \rightarrow \infty$. This latter property was extended in [8] to the more general case of unknown colored noise plus multiple sinusoidal signals. In all these and other works, however, no distribution limit theorems of the $\operatorname{AR}(p)$ spectral estimator for a general mixed spectrum process containing multiple sinusoids plus arbitrary colored noise were developed.

Recently, a first effort in response to this need was pursued in [9]. That effort relied on the limiting distribution of the sample autocovariances given in [10]. In this paper, we rely on that result to develop a more concise set of limiting distributions regarding the finite-order AR modeling of a mixed spectrum process. We begin by summarizing the basic background of such a process and AR modeling in Section II. In Section III, the asymptotic normality of the coefficients and innovations variance of the $\operatorname{AR}(p)$ model are derived. The limiting distribution of the corresponding $\operatorname{AR}(p)$ spectral estimator is derived in Section IV. Numerical simulations are given in Section V in order to illustrate the accuracy of the analytical results. Finally, Section VI contains a brief summary of results, a discussion of their practical value in all too common realistic and important settings, and suggestions for future work. The proofs of the results presented in this paper are provided in the Appendix.

\section{PRELIMINARIES}

In this work, a mixed spectrum process is defined as $y_{n}=$ $x_{n}+\varepsilon_{n}$, where

$$
\begin{aligned}
& x_{n}=\sum_{m=1}^{q} A_{m} \cos \left(\omega_{m} n+\phi_{m}\right) \\
& \varepsilon_{n}=\sum_{j=-\infty}^{\infty} \psi_{j} \zeta_{n-j}
\end{aligned}
$$

where

$$
\begin{aligned}
\left\{\zeta_{n}\right\}_{n=-\infty}^{\infty} & \sim \text { i.i.d. }\left(0, \sigma_{\zeta}^{2}\right) \\
E\left\{\zeta_{n}^{4}\right\} & =\kappa \sigma_{\zeta}^{4}<\infty \\
\sum\left|\psi_{j}\right| & <\infty
\end{aligned}
$$

$\left\{A_{m}\right\}_{m=1}^{q}$ and $\left\{\omega_{m}\right\}_{m=1}^{q} \in(0, \pi)$ are unknown amplitudes and frequencies, and where $\left\{\phi_{m}\right\}_{m=1}^{q} \sim$ i.i.d. U[ $[-\pi, \pi]$. Assuming 
$\left\{\phi_{m}\right\}_{m=1}^{q}$ and $\left\{\zeta_{n}\right\}$ are independent, the theoretical autocovariance function of $y_{n}$ is given by $r_{\tau}^{y}=r_{\tau}^{x}+r_{\tau}^{\varepsilon}$, where

$$
\begin{aligned}
& r_{\tau}^{x}=\sum_{m=1}^{q} \frac{A_{m}^{2}}{2} \cos \left(\omega_{m} \tau\right) \\
& r_{\tau}^{\varepsilon}=\sigma_{\zeta}^{2} \sum_{j=-\infty}^{\infty} \psi_{j} \psi_{j+\tau}
\end{aligned}
$$

are the autocovariance functions of $x_{n}$ and $\varepsilon_{n}$, respectively. The $p$ th-order $\operatorname{AR}$ (i.e., $\operatorname{AR}(p))$ predictor of $y_{n}$ is

$$
\hat{y}_{n}=-\sum_{k=1}^{p} a_{k} \cdot y_{n-k}
$$

with the prediction error variance $E\left\{\left(\hat{y}_{n}-y_{n}\right)^{2}\right\}=\sigma_{p}^{2}$. The minimum variance parameters $\boldsymbol{a}_{p}^{\prime}=\left[a_{1}, a_{2}, \ldots, a_{p}\right]$ and $\sigma_{p}^{2}$, which minimize this prediction error variance, are readily obtained from the Yule-Walker (also termed minimum variance) equations (see [1] or [3])

$$
\begin{aligned}
& \boldsymbol{a}_{p}=-\boldsymbol{R}_{y}^{-1} \cdot \boldsymbol{r}^{y} \\
& \sigma_{p}^{2}=r_{0}^{y}+\boldsymbol{r}^{y^{\prime}} \cdot \boldsymbol{a}_{p} .
\end{aligned}
$$

Here, the $(p \times p)$ Toeplitz matrix $\boldsymbol{R}_{y}$ and the $(p \times 1)$ vector $\boldsymbol{r}^{y}$ are given by

$$
\boldsymbol{R}_{y} \triangleq\left[\begin{array}{cccc}
r_{0}^{y} & r_{1}^{y} & \cdots & r_{p-1}^{y} \\
r_{1}^{y} & r_{0}^{y} & \cdots & r_{p-2}^{y} \\
\vdots & \vdots & \cdots & \vdots \\
r_{p-1}^{y} & r_{p-2}^{y} & \cdots & r_{0}^{y}
\end{array}\right] \quad \text { and } \quad \boldsymbol{r}^{y} \triangleq\left[\begin{array}{c}
r_{1}^{y} \\
r_{2}^{y} \\
\vdots \\
r_{p}^{y}
\end{array}\right]
$$

The least squares estimators $\hat{\boldsymbol{a}}_{p}$ and $\hat{\sigma}_{p}^{2}$ of (1) based on a set of $N$ samples can be obtained by replacing $r_{\tau}^{y}$ with the sample autocovariance function defined by

$$
\hat{r}_{\tau}^{y}=\frac{1}{N} \sum_{n=1}^{N-\tau} y_{n} \cdot y_{n+\tau}
$$

Denote by $\hat{\boldsymbol{R}}_{y}$ and $\hat{\boldsymbol{r}}^{y}$ the sample versions of $\boldsymbol{R}_{y}$ and $\boldsymbol{r}^{y}$, respectively. We then have

$$
\begin{gathered}
\hat{\boldsymbol{a}}_{p}=-\hat{\boldsymbol{R}}_{y}^{-1} \cdot \hat{\boldsymbol{r}}^{y} \\
\hat{\sigma}_{p}^{2}=\hat{\boldsymbol{r}}_{0}^{y}+\hat{\boldsymbol{r}}^{y^{\prime}} \cdot \hat{\boldsymbol{a}}_{p} .
\end{gathered}
$$

Let $S_{p}(\omega)$ denote the minimum variance $\operatorname{AR}(p)$ spectrum and $\hat{S}_{p}(\omega)$ denote its least squares estimator. Then

$$
\begin{aligned}
S_{p}(\omega) & =\frac{\sigma_{p}^{2}}{\left|\sum_{k=0}^{p} a_{k} e^{-i k \omega}\right|^{2}} \triangleq \frac{\sigma_{p}^{2}}{\left|\rho_{p}(\omega)\right|^{2}} \\
\hat{S}_{p}(\omega) & =\frac{\hat{\sigma}_{p}^{2}}{\left|\sum_{k=0}^{p} \hat{a}_{k} e^{-i k \omega}\right|^{2}} \triangleq \frac{\hat{\sigma}_{p}^{2}}{\left|\hat{\rho}_{p}(\omega)\right|^{2}}
\end{aligned}
$$

where $\hat{a}_{0}=a_{0}=1$. The goal of this paper is to arrive at the large sample distribution of (3b). In particular, we desire to characterize its statistical behavior in the frequency regions near the point spectrum.

\section{Limiting Distributions of $\operatorname{AR}(p)$ PARAmeters}

In this section, the limiting distributions of $\hat{\boldsymbol{a}}_{p}$ and $\hat{\sigma}_{p}^{2}$ defined by (2) are derived using a relatively recent result concerning the limiting distribution of the sample autocovariance function $\hat{r} y$.

The large sample distribution of the sample autocovariance function $\hat{r}{ }_{\tau}^{y}$ for the case of a single sinusoid plus white noise was recently derived in [7]. More recently, [10] extended the result to the more general setting of multiple sinusoids plus colored noise with continuous spectrum. Their main result is given in Theorem 1. First define the following quantities:

$$
\begin{aligned}
\boldsymbol{r}_{p}^{y} & =\left[\begin{array}{lll}
r_{0}^{y} & r_{1}^{y} \cdots & r_{p}^{y}
\end{array}\right]^{\prime} \\
\boldsymbol{r}_{p}^{\varepsilon} & =\left[\begin{array}{lll}
r_{0}^{\varepsilon} & r_{1}^{\varepsilon} \cdots r_{p}^{\varepsilon}
\end{array}\right]^{\prime}
\end{aligned}
$$

where $(\cdot)^{\prime}$ denotes vector transposition. Notice that $\boldsymbol{r}_{p}^{y}=$ $\left[r_{0}^{y}, \boldsymbol{r}^{y^{\prime}}\right]^{\prime}$.

Theorem 1 [10]: Given a sample of size $N$ of $y_{n}$, and the assumptions and quantities described in Section II, then $\sqrt{N}\left(\hat{\boldsymbol{r}}_{p}^{y}-\boldsymbol{r}_{p}^{y}\right)$ is asymptotically normal with zero mean and covariance matrix $\Sigma=\left[\sigma_{j k}\right]_{j, k=0, \ldots, p}$ given by

$$
\begin{aligned}
\sigma_{j k}= & \sum_{m=1}^{q} 2 A_{m}^{2} \cos \left(j \omega_{m}\right) \cos \left(k \omega_{m}\right) \cdot \sum_{\tau=-\infty}^{\infty} r_{\tau}^{\varepsilon} \cos \left(\omega_{m} \tau\right) \\
& +(\kappa-3) r_{j}^{\varepsilon} r_{k}^{\varepsilon}+\sum_{\tau=-\infty}^{\infty}\left\{r_{\tau}^{\varepsilon} r_{\tau+j-k}^{\varepsilon}+r_{\tau+j}^{\varepsilon} r_{\tau-k}^{\varepsilon}\right\}
\end{aligned}
$$

Theorem 1 is a generalization of the corresponding result in [1] for a regular process. The influence of point spectrum is reflected in the first term on the right of (4).

The large sample distribution of $\hat{\boldsymbol{a}}_{p}$ is given in the next theorem. Because it is done in a mixed spectrum setting, it is an extension of results such as those in [1]-[3].

Theorem 2: With $\boldsymbol{a}_{p}$ and $\hat{\boldsymbol{a}}_{p}$ defined as in (1a) and (2a), we have

$$
\sqrt{N}\left(\hat{\boldsymbol{a}}_{p}-\boldsymbol{a}_{p}\right)=-\boldsymbol{R}_{y}^{-1} \cdot \boldsymbol{A} \cdot \sqrt{N}\left(\hat{\boldsymbol{r}}_{p}^{y}-\boldsymbol{r}_{p}^{y}\right)+o_{p}(1) .
$$

Thus,

$$
\sqrt{N}\left(\hat{\boldsymbol{a}}_{p}-\boldsymbol{a}_{p}\right) \stackrel{d}{\longrightarrow} \mathrm{N}\left(\mathbf{0}, \boldsymbol{R}_{y}^{-1} \boldsymbol{A} \boldsymbol{\Sigma} \boldsymbol{A}^{\prime} \boldsymbol{R}_{y}^{-1}\right)
$$

where

$$
\begin{aligned}
& A \triangleq\left[\begin{array}{cccccc}
a_{1} & a_{2} & a_{3} & \cdots & a_{p} & 0 \\
a_{2} & a_{3} & a_{4} & \cdots & 0 & 0 \\
a_{3} & a_{4} & a_{5} & \cdots & 0 & 0 \\
\vdots & \vdots & \vdots & \cdots & \vdots & \vdots \\
a_{p-1} & a_{p} & 0 & \cdots & 0 & 0 \\
a_{p} & 0 & 0 & \cdots & 0 & 0
\end{array}\right] \\
& +\left[\begin{array}{cccccc}
0 & a_{0} & 0 & \cdots & 0 & 0 \\
0 & a_{1} & a_{0} & \cdots & 0 & 0 \\
0 & a_{2} & a_{1} & \cdots & 0 & 0 \\
\vdots & \vdots & \vdots & \cdots & \vdots & \vdots \\
0 & a_{p-2} & a_{p-3} & \cdots & a_{0} & 0 \\
0 & a_{p-1} & a_{p-2} & \cdots & a_{1} & a_{0}
\end{array}\right] .
\end{aligned}
$$


The matrix $\boldsymbol{A}$ in Theorem 2 has a special property. From the Yule-Walker equation (1a), we know that

$$
\left[\begin{array}{ll}
\boldsymbol{r}^{y} & \boldsymbol{R}_{y}
\end{array}\right] \cdot\left[\begin{array}{c}
1 \\
\boldsymbol{a}_{p}
\end{array}\right]=\mathbf{0}
$$

so that the following equality can be easily deduced:

$$
\left[\begin{array}{cccc}
r_{1}^{y} & r_{0}^{y} & \cdots & r_{p-1}^{y} \\
r_{2}^{y} & r_{1}^{y} & \cdots & r_{p-2}^{y} \\
\vdots & \vdots & \cdot & \vdots \\
r_{p}^{y} & r_{p-1}^{y} & \cdots & r_{0}^{y}
\end{array}\right] \cdot\left[\begin{array}{c}
1 \\
a_{1} \\
\vdots \\
a_{p}
\end{array}\right]=\mathbf{0}=\boldsymbol{A} \cdot\left[\begin{array}{c}
r_{0}^{y} \\
r_{1}^{y} \\
\vdots \\
r_{p}^{y}
\end{array}\right]=\boldsymbol{A} \cdot \boldsymbol{r}_{p}^{y}
$$

Next, we derive the limiting distribution of $\hat{\sigma}_{p}^{2}$. This will require the following lemma.

Thus,

Lemma 1: $\sqrt{N}\left(\hat{\boldsymbol{r}}^{y^{\prime}} \hat{\boldsymbol{a}}_{p}-\boldsymbol{r}^{y^{\prime}} \boldsymbol{a}_{p}\right)=\boldsymbol{\beta}^{\prime} \sqrt{N}\left(\hat{\boldsymbol{r}}_{p}^{y}-\boldsymbol{r}_{p}^{y}\right)+o_{p}(1)$.

$$
\sqrt{N}\left(\hat{\boldsymbol{r}}^{y^{\prime}} \hat{\boldsymbol{a}_{p}}-\boldsymbol{r}^{y^{\prime}} \boldsymbol{a}_{p}\right) \stackrel{d}{\longrightarrow} \mathrm{N}\left(\mathbf{0}, \boldsymbol{\beta}^{\prime} \boldsymbol{\Sigma} \boldsymbol{\beta}\right)
$$

where $\boldsymbol{\beta}^{\prime}=\left[g_{0}^{a}-12 g_{1}^{a} \cdots 2 g_{p}^{a}\right]$, and where $g_{\tau}^{a}$ is the convolution of $\left[\ldots, 0, a_{0}, \ldots, a_{p}, 0, \ldots\right]$ with its reverse at lag $\tau$; specifically

$$
g_{i}^{a}=\sum_{j=0}^{p-i} a_{j} a_{j+i}=\sum_{j=\infty}^{\infty} a_{j} a_{j+i}
$$

with $a_{0} \equiv 1, a_{i}=0$ for $i<0$ and $i>p$.

Remark 1: Defining $\boldsymbol{\nu}=\left[g_{0}^{a} 2 g_{1}^{a} \cdots 2 g_{p}^{a}\right]$, then $\boldsymbol{\nu}^{\prime} \boldsymbol{\gamma}(\omega)=$ $\left|\rho_{p}(\omega)\right|^{2}$, where

$$
\gamma(\omega)^{\prime}=[1 \cos (\omega) \cos (2 \omega) \cdots \cos (p \omega)] .
$$

To see this, first recall that the correlation of two real-valued sequences $w_{n}$ and $v_{n}$ is equivalent to $g_{\tau}^{w v}=w_{n} \star v_{-n}$, where $\star$ denotes convolution. Denote by $W(\omega)$ and $V(\omega)$ the Fourier transforms of $w_{n}$ and $v_{n}$, respectively. The Fourier transform of $g_{\tau}^{w v}$ is

$$
F\left\{g_{\tau}^{w \vartheta}\right\}=W(\omega) V(-\omega)=W(\omega) V^{*}(\omega) .
$$

The second equality follows because $F\left(v_{-n}\right)=V(-\omega)=$ $V^{*}(\omega)$ for real-valued sequence $v_{n}$. Finally, observe that

$$
\begin{aligned}
\nu^{\prime} \gamma(\omega) & =g_{0}^{a}+2 g_{1}^{a} \cos (\omega)+\cdots+2 g_{p}^{a} \cos (p \omega) \\
& =\sum_{\tau=\infty}^{\infty} g_{\tau}^{a} e^{-i \tau \omega}=F\left\{g_{\tau}^{a}\right\}=\rho_{p}(\omega) \rho_{p}^{*}(\omega) \\
& =\left|\rho_{p}(\omega)\right|^{2} .
\end{aligned}
$$

Using Lemma 1, Remark 1, (1b), and (2b), we arrive at the following large sample distribution for $\hat{\sigma}_{p}^{2}$.

Theorem 3: With $\sigma_{p}^{2}$ and $\hat{\sigma}_{p}^{2}$ defined as in (1b) and (2b), we have the following asymptotic normality:

$$
\sqrt{N}\left(\hat{\sigma}_{p}^{2}-\sigma_{p}^{2}\right) \stackrel{d}{\longrightarrow} N\left(0, \Psi_{\hat{\sigma}_{p}^{2}}\right)
$$

where the time-domain expression for $\Psi_{\hat{\sigma}_{p}^{2}}$ is

$$
\Psi_{\hat{\sigma}_{p}^{2}}=\nu^{\prime} \Sigma \nu
$$

Equation (7) is in quadratic form involving the covariance matrix $\boldsymbol{\Sigma}$. Since the expressions for the elements of $\boldsymbol{\Sigma}$, (4), involve infinite summations, a direct time-domain interpretation and simplification of $\Psi_{\hat{\sigma}_{p}^{2}}$ is difficult. Our next result is an equivalent frequency-domain expression for $\boldsymbol{\Sigma}$ which provides more insight into the influence of point spectrum on an AR spectral estimator, and leads to a frequency-domain expression for $\Psi_{\hat{\sigma}_{p}^{2}}$, as given in Corollary 1. The frequency-domain expression for the covariance matrix $\boldsymbol{\Sigma}$ of the limiting distribution of $\sqrt{N}\left(\hat{\boldsymbol{r}}_{p}-\boldsymbol{r}_{p}\right)$ in (4) is given by

$$
\begin{aligned}
\Sigma= & \frac{2}{2 \pi} \int_{-\pi}^{\pi}\left\{\left[2 S_{x}(\omega)+S_{\epsilon}(\omega)\right] S_{\epsilon}(\omega) \boldsymbol{\Gamma}(\omega)\right\} d \omega \\
& +(\kappa-3)\left\{\frac{1}{2 \pi} \int_{-\pi}^{\pi} S_{\epsilon}(\omega) \boldsymbol{\gamma}(\omega) d \omega\right\} \\
& \cdot\left\{\frac{1}{2 \pi} \int_{-\pi}^{\pi} S_{\epsilon}(\omega) \boldsymbol{\gamma}(\omega)^{\prime} d \omega\right\}
\end{aligned}
$$

where

$$
\begin{aligned}
S_{x}(\omega) & =2 \pi \sum_{m=1}^{q} \frac{A_{m}^{2}}{4} \cdot \delta\left(\omega \pm \omega_{m}\right) \\
S_{\epsilon}(\omega) & =\sum_{\tau=-\infty}^{\infty} r_{\tau}^{\epsilon} \cdot e^{-i \tau \omega} \\
\boldsymbol{\Gamma}(\omega) & =\gamma(\omega) \cdot \gamma(\omega)^{\prime} \\
\gamma(\omega)^{\prime} & =[1 \cos (\omega) \cos (2 \omega) \cdots \cos (p \omega)] .
\end{aligned}
$$

Here, $\delta(\cdot)$ is the Dirac delta function. The same frequency-domain expression as the first term on the right-hand side of (8) is given in [11] where the case of more manageable complex sinusoids is addressed. Consequently, (8) is not only an extension to the case of real sinusoids, but also includes a frequency-domain expression for the rightmost term in (8); an expression not provided in [11]. It leads immediately to the following corollary.

Corollary 1: The frequency-domain expression of $\Psi_{\hat{\sigma}_{p}^{2}}$ is given by

$$
\begin{gathered}
\Psi_{\hat{\sigma}_{p}^{2}}=\frac{2}{2 \pi} \int_{-\pi}^{\pi}\left\{\left[2 S_{x}(\omega)+S_{\epsilon}(\omega)\right] S_{\epsilon}(\omega)\left|\rho_{p}(\omega)\right|^{4}\right\} d \omega \\
+(\kappa-3)\left\{\frac{1}{2 \pi} \int_{-\pi}^{\pi} S_{\epsilon}(\omega)\left|\rho_{p}(\omega)\right|^{2} d \omega\right\}^{2} .
\end{gathered}
$$

Theorem 3 is, to our knowledge, the first of its kind which states that for a general mixed spectrum process $\hat{\sigma}_{p}^{2}$ is asymptotically unbiased estimator for $\sigma_{p}^{2}$. For the case of Gaussian noise (i.e., $\kappa=3$ ), Corollary 1 states that the point spectrum influence is a combination of a direct influence on only the discrete frequencies $\left\{\omega_{m}\right\}_{m=1}^{q}$, plus an indirect influence on the entire frequency range via $\left|\rho_{p}(\omega)\right|^{2}$.

\section{Limiting Distribution of AR( $p$ ) Spectral EstimatoR}

The results of the last section for the AR parameters set the stage for our goal of this paper, which is the large sample approximate distribution of AR spectral estimator ( $3 b$ ) for the case 
of a mixed spectrum process. To this end, we first describe the limiting distribution of $\left|\hat{\rho}_{p}(\omega)\right|^{2}$.

Lemma 2:

$$
\begin{aligned}
\sqrt{N}\left[\left|\hat{\rho}_{p}(\omega)\right|^{2}-\right. & \left.\left|\rho_{p}(\omega)\right|^{2}\right] \\
& =2[\boldsymbol{A} \boldsymbol{\gamma}(\omega)]^{\prime} \cdot \sqrt{N}\left(\hat{\boldsymbol{a}_{p}}-\boldsymbol{a}_{p}\right)+o_{p}(1) .
\end{aligned}
$$

Thus,

$$
\sqrt{N}\left[\left|\hat{\rho}_{p}(\omega)\right|^{2}-\left|\rho_{p}(\omega)\right|^{2}\right] \stackrel{d}{\longrightarrow} \mathrm{N}\left(0, \Psi_{\left|\hat{\rho}_{p}\right|^{2}}\right)
$$

where

$$
\Psi_{\left|\hat{\rho}_{p}\right|^{2}}=\Psi_{\left|\hat{\rho}_{p}(\omega)\right|^{2}}=4[\boldsymbol{A} \boldsymbol{\gamma}(\omega)]^{\prime} \boldsymbol{R}_{y}^{-1} \boldsymbol{A} \boldsymbol{\Sigma} \boldsymbol{A}^{\prime} \boldsymbol{R}_{y}^{-1}[\boldsymbol{A} \boldsymbol{\gamma}(\omega)] .
$$

We now give the main result of this paper.

Theorem 4: $\sqrt{N}\left[\hat{S}_{p}(\omega)-S_{p}(\omega)\right] \stackrel{d}{\longrightarrow} \mathrm{N}\left(0, \Psi_{\hat{S}_{p}}\right)$, where

$$
\begin{aligned}
& \Psi_{\hat{S}_{p}}= \frac{1}{\sigma_{p}^{4}} S_{p}^{2}(\omega)\left\{\Psi_{\hat{\sigma}_{p}^{2}}+S_{p}^{2}(\omega) \cdot \Psi_{\left|\hat{\rho}_{p}\right|^{2}}\right. \\
&\left.-2 S_{p}(\omega) \cdot \Psi_{\hat{\sigma}_{p}^{2},\left|\hat{\rho}_{p}\right|^{2}}\right\} \\
& \Psi_{\hat{\sigma}_{p}^{2}}= \boldsymbol{\nu}^{\prime} \boldsymbol{\Sigma} \boldsymbol{\nu} \\
& \Psi_{\left|\hat{\rho}_{p}\right|^{2}}=4[\boldsymbol{A} \gamma(\omega)]^{\prime} \boldsymbol{R}_{y}^{-1} \boldsymbol{A} \boldsymbol{\Sigma} \boldsymbol{A}^{\prime} \boldsymbol{R}_{y}^{-1}[\boldsymbol{A} \boldsymbol{\gamma}(\omega)] \\
& \Psi_{\hat{\sigma}_{p}^{2},\left|\hat{\rho}_{p}\right|^{2}}=-2 \boldsymbol{\nu}^{\prime} \boldsymbol{\Sigma} \boldsymbol{A}^{\prime} \boldsymbol{R}_{y}^{-1} \boldsymbol{A} \boldsymbol{\gamma}(\omega) .
\end{aligned}
$$

Theorem 4 is a new result that provides a complete statistical description of the large sample $\operatorname{AR}(p)$ spectral estimator for a general mixed spectrum process. An expression for the asymptotic variance of $\hat{S}_{p}(\omega)$ was given in [6], but no limiting distribution was given. Theorem 4 establishes the asymptotic normality of $\hat{S}_{p}(\omega)$. It would be desirable to simplify the variance expression of Theorem 4 in order to gain deeper insight into the influence of factors such as the shape of the noise color and the signal-to-noise ratio (SNR). To this end, we have investigated the combined use of (8) and properties of circulant approximants of quadratic forms involving covariance information associated with AR processes [12]. To date, we have had limited success.

Recall from [7] and [8] that the theoretical $\operatorname{AR}(p)$ approximant converges to $+\infty$ at tone frequencies $\left\{\omega_{m}\right\}_{m=1}^{q}$ as $p \rightarrow$ $\infty$. This is not surprising since sinusoids have point mass. It is this same property that is responsible for the convergence of the $p$ th-order minimum variance (MV), denoted $\mathrm{MV}(p)$ spectrum at point frequency to the process power spectrum as $p \rightarrow \infty$ [8], [13]. The $\operatorname{MV}(p)$ spectrum is defined to be the inverse of the sum of $\operatorname{AR}(q)$ spectra for $q \leq p$. Since the family of MV spectra have demonstrated significant potential for identifying point spectrum with arbitrary noise, and since the $\mathrm{MV}(p)$ spectrum is related to the $\operatorname{AR}(k)$ spectra for $k \leq p$, a valid question in the context of this paper is: What is the statistical behavior of the $\operatorname{AR}(p)$ spectral estimator as $p \rightarrow \infty$. Clearly, this question requires conditions on $p / N$ as both $p$ and $N \rightarrow \infty$. This problem was studied in [5] for regular processes, wherein it was proved that if $p, N \rightarrow \infty$ such that $p^{3} / N \rightarrow 0$, then $\sqrt{N / p}\left[\left(\hat{S}_{p}-S_{p}\right) / S_{p}\right]$ converges to a normal distribution with the asymptotic variance equals 4 if $\omega=0, \pi$, and 2 otherwise. We conclude this section with the following conjecture.
Conjecture 1: Define $\Psi_{\hat{S}_{p}}$ as in Theorem 4, then

$$
\begin{aligned}
& \lim _{p \rightarrow \infty} \frac{1}{p S_{p}^{2}(\omega)} \Psi_{\hat{S}_{p}} \\
& \rightarrow \begin{cases}2, & \text { if } \omega \neq 0, \pi \text { and } \omega \notin\left\{w_{m}\right\}_{m=1}^{q} \\
4, & \text { if } \omega=0, \pi \text { and } \omega \notin\left\{w_{m}\right\}_{m=1}^{q} \\
M, & \text { if } \omega \in\left\{w_{m}\right\}_{m=1}^{q}\end{cases}
\end{aligned}
$$

where $M$ is some constant. Even though in the setting of [5] there is no point spectrum, given the orthogonalizing property of a frequency decomposition of a correlation function, it is not surprising that (13) approaches the value of 2 at $\omega \notin\left\{w_{m}\right\}_{m=1}^{q}$. After numerous unsuccessful attempts to prove this, we have decided to let it remain as a conjecture, as did Sakai [6]. This conjecture was arrived at by investigating a number of simulations. The unknown value $M$ in (13) is of particular interest with respect to MV type of spectral analysis of mixed processes proposed in [8]. To illustrate this convergent property, consider the process $y_{n}=x_{n}+\varepsilon_{n}$, where

$$
\begin{aligned}
x_{n} & =\cos \left(\frac{n \pi}{4}\right) \\
\varepsilon_{n} & =0.3 \varepsilon_{n-1}-0.9 \varepsilon_{n-2}+\zeta_{n} \\
\zeta_{n} & \sim \text { i.i.d. } \mathrm{N}(0,1) .
\end{aligned}
$$

Fig. 1 shows the value of $\left(1 / p S_{p}^{2}(\omega)\right) \Psi_{\hat{S}_{p}}$ for

$$
p=[5,10,30,50,100,200,500]
$$

over the frequency range $[0, \pi]$. At tone frequency $\omega=\pi / 4$, this numerical approximation shows that the normalized variance of the AR spectrum remains bounded.

\section{NUMERICAL ANALYSIS}

The proofs of the results in the previous sections relied on the delta method, which is a first-order Taylor series approximation approach. As such, it is important to investigate the accuracy of this approximation. This investigation could proceed by a theoretical analysis of the remainder of the approximation. But such an approach would be nontrivial to say the very least. For this reason, and to illustrate its potential in a more user-friendly fashion, our investigation will proceed in the context of an example.

In this section, we will illustrate the potential accuracy of our results by fitting an $\operatorname{AR}(p)$ model to the mixed spectrum process $y_{n}=x_{n}+\varepsilon_{n}$, where

$$
\begin{aligned}
x_{n} & =\cos \left(\frac{n \pi}{2}\right)+\cos \left(\frac{3 n \pi}{4}\right) \\
\varepsilon_{n} & =0.3 \varepsilon_{n-1}-0.9 \varepsilon_{n-2}+\zeta_{n} \\
\zeta_{n} & \sim \text { i.i.d. } \mathrm{N}(0,1) .
\end{aligned}
$$

Each of the following two cases to be considered utilized 1000 random simulations of $y_{n}$. The numerical results are compared to the analytical results for each case, emphasizing only the first four elements the coefficient vector $\hat{\boldsymbol{a}}_{p}$, and the innovation variance $\hat{\sigma}_{p}^{2}$. Case 1 and Case 2 consider record lengths $N=500$ and 1000 of $y_{n}$, respectively. The SNR for both cases 


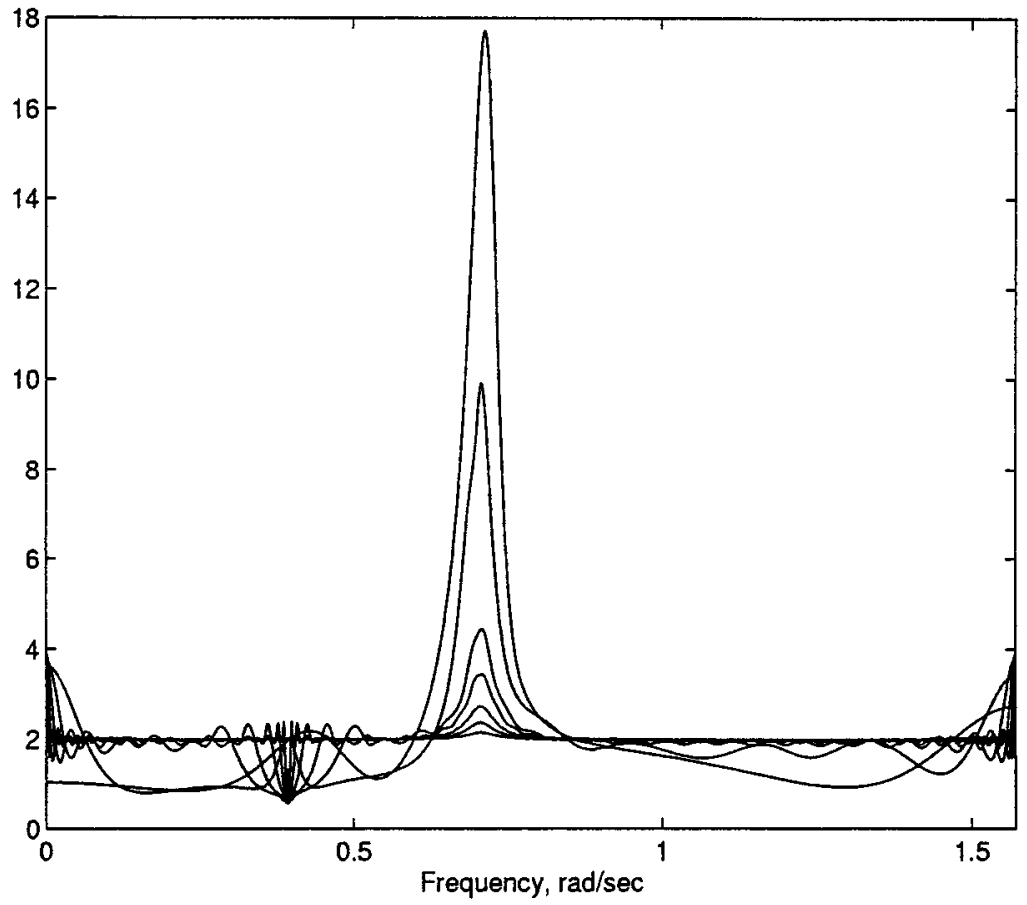

Fig. 1. Numerical approximation of (13) for $p=[5,10,30,50,100,200,500]$.

TABLE I

CASE 1: SNR $=-7.3221 \mathrm{~dB}, N=500$

\begin{tabular}{|c|c|c|c|c|c|c|c|}
\hline \multirow{2}{*}{$\begin{array}{c}\text { AR order } \\
p \\
\end{array}$} & \multicolumn{2}{|c|}{ Innovations variance, $\hat{\sigma}^{2}$} & \multicolumn{5}{|c|}{ AR coefficients, $\hat{\boldsymbol{a}}$} \\
\hline & $E\left\{\hat{\sigma}^{2}\right\}$ & $N \cdot \operatorname{Var}\left\{\hat{\sigma}^{2}\right\}$ & $E\{\hat{a}\}$ & & $N \cdot l$ & $r\{\hat{\boldsymbol{a}}\}$ & \\
\hline \multirow{5}{*}{5} & \multirow{5}{*}{$\begin{array}{c}1.6246 \\
(1.6411)\end{array}$} & \multirow{5}{*}{$\begin{array}{c}5.4257 \\
(5.4159)\end{array}$} & $\begin{array}{c}0.0943 \\
(0.0916)\end{array}$ & $\begin{array}{c}0.5598 \\
(0.4968)\end{array}$ & $\begin{array}{c}0.1934 \\
(0.1429)\end{array}$ & $\begin{array}{c}0.4469 \\
(0.3699)\end{array}$ & $\begin{array}{c}0.1664 \\
(0.1431)\end{array}$ \\
\hline & & & 0.8156 & 0.1934 & 0.6124 & 0.4359 & 0.3073 \\
\hline & & & $(0.8154)$ & $(0.1429)$ & $(0.5245)$ & $(0.3708)$ & $(0.2634)$ \\
\hline & & & $\begin{array}{c}-0.0153 \\
(-0.0159)\end{array}$ & $\begin{array}{c}0.4469 \\
(0.3699)\end{array}$ & $\begin{array}{c}0.4359 \\
(0.3708)\end{array}$ & $\begin{array}{c}0.8286 \\
(0.7291)\end{array}$ & $\begin{array}{c}0.3926 \\
(0.3682)\end{array}$ \\
\hline & & & $\begin{array}{c}0.1627 \\
(0.1592)\end{array}$ & $\begin{array}{c}0.1664 \\
(0.1431)\end{array}$ & $\begin{array}{c}0.3073 \\
(0.2634)\end{array}$ & $\begin{array}{c}0.3926 \\
(0.3682)\end{array}$ & $\begin{array}{c}0.4420 \\
(0.4098)\end{array}$ \\
\hline \multirow{5}{*}{50} & \multirow{5}{*}{$\begin{array}{c}0.9450 \\
(1.0616)\end{array}$} & \multirow{5}{*}{$\begin{array}{c}2.9171 \\
(2.3039)\end{array}$} & $\begin{array}{c}-0.1595 \\
(-0.1604)\end{array}$ & $\begin{array}{c}1.0752 \\
(0.9241)\end{array}$ & $\begin{array}{c}-0.0684 \\
(-0.0523)\end{array}$ & $\begin{array}{c}0.9644 \\
(0.8164)\end{array}$ & $\begin{array}{c}-0.0775 \\
(-0.0493)\end{array}$ \\
\hline & & & $\begin{array}{c}0.8979 \\
(0.9133)\end{array}$ & $\begin{array}{c}-0.0684 \\
(-0.2723)\end{array}$ & $\begin{array}{c}0.9903 \\
(1.0593)\end{array}$ & $\begin{array}{c}-0.1442 \\
(-0.5050)\end{array}$ & $\begin{array}{c}0.9899 \\
(0.9000)\end{array}$ \\
\hline & & & -0.0146 & 1.3496 & -0.7959 & 2.6297 & -0.7600 \\
\hline & & & $\frac{(-0.0093)}{0.0525}$ & $(0.9073)$ & $\frac{(-0.505)}{0.0014}$ & $(1.8900)$ & $(-0.5181)$ \\
\hline & & & $(0.0462)$ & $(0.0258)$ & $(0.9000)$ & $(-0.5181)$ & $(1.9489)$ \\
\hline
\end{tabular}

is $-7.3221 \mathrm{~dB}$, and AR models of order $p=5$ and 50 are considered. The simulation and theoretical results are tabulated in Tables I and II for Cases 1 and 2, respectively. The data in parentheses correspond to the theoretical results. An interpretation of these data shows an excellent match between the simulation statistics and theoretical results. A slight deviation can be observed for the cases where $p=50$. This is due to the decrease in the statistical reliability of the autocovariance estimates needed for solving the Yule-Walker equations (2); specifically, to the increased number of needed parameter estimates for the given sample size.

To gain a visual appreciation for the accuracy of the results, the estimated means and variances of $\hat{S}(\omega)$ (dashed line) are plotted against the theoretical results (solid line) for Case 1, as given in Fig. 2. In particular, the accuracy of the spectral mean and variance expressions of Theorem 4 is very high in the neighborhood of the point spectrum.

\section{CONCLUSION}

This work generalized the conventional large sample properties of an AR estimator for a regular process with an unspecified continuous spectrum to those for a stochastic process containing a mixed spectrum. In the process, asymptotic normality of the AR coefficients, innovation variances, and AR spectral estimate of a finite-order autoregression were derived. Numerical simulations demonstrated the reliability of the analytical results. The well-known statistics of the AR spectral estimator for regular 
TABLE II

CASE 2: SNR $=-7.3221 \mathrm{~dB}, N=1000$

\begin{tabular}{|c|c|c|c|c|c|c|c|}
\hline \multirow{2}{*}{$\begin{array}{c}\mathrm{AR} \text { order } \\
p\end{array}$} & \multicolumn{2}{|c|}{ Innovations variance, $\hat{\sigma}^{2}$} & \multicolumn{5}{|c|}{ AR coefficients, $\hat{a}$} \\
\hline & $E\left\{\hat{\sigma}^{2}\right\}$ & $N \cdot \operatorname{Var}\left\{\hat{\sigma}^{2}\right\}$ & $E\{\hat{a}\}$ & & $N \cdot V$ & $r\{\hat{\boldsymbol{a}}\}$ & \\
\hline \multirow{4}{*}{5} & \multirow{4}{*}{$\begin{array}{c}1.6383 \\
(1.6411)\end{array}$} & \multirow{4}{*}{$\begin{array}{c}6.0849 \\
(5.4195)\end{array}$} & $\begin{array}{c}0.0917 \\
(0.0916)\end{array}$ & $\begin{array}{c}0.5353 \\
(0.4968)\end{array}$ & $\begin{array}{c}0.1598 \\
(0.1429)\end{array}$ & $\begin{array}{c}0.4016 \\
(0.3699)\end{array}$ & $\begin{array}{c}0.1405 \\
(0.1431)\end{array}$ \\
\hline & & & $\begin{array}{c}0.8144 \\
(0.8154)\end{array}$ & $\begin{array}{c}0.1598 \\
(0.1429)\end{array}$ & $\begin{array}{c}0.5406 \\
(0.5245)\end{array}$ & $\begin{array}{c}0.3859 \\
(0.3708)\end{array}$ & $\begin{array}{c}0.2684 \\
(0.2634)\end{array}$ \\
\hline & & & $\begin{array}{c}-0.0153 \\
(-0.0159)\end{array}$ & $\begin{array}{c}0.4016 \\
(0.3699)\end{array}$ & $\begin{array}{c}0.3859 \\
(0.3708)\end{array}$ & $\begin{array}{c}0.7783 \\
(0.7291)\end{array}$ & $\begin{array}{c}0.3799 \\
(0.3682)\end{array}$ \\
\hline & & & $\begin{array}{c}0.1593 \\
(0.1592)\end{array}$ & $\begin{array}{c}0.1405 \\
(0.1431)\end{array}$ & $\begin{array}{c}0.2684 \\
(0.2634)\end{array}$ & $\begin{array}{c}0.3799 \\
(0.3682)\end{array}$ & $\begin{array}{c}0.4229 \\
(0.4098)\end{array}$ \\
\hline \multirow{4}{*}{50} & \multirow{4}{*}{$\begin{array}{c}1.0070 \\
(1.0616)\end{array}$} & \multirow{4}{*}{$\begin{array}{c}2.4638 \\
(2.3039)\end{array}$} & $\begin{array}{c}-0.1610 \\
(-0.1604)\end{array}$ & $\begin{array}{c}1.0429 \\
(0.9241)\end{array}$ & $\begin{array}{c}-0.0927 \\
(-0.0523)\end{array}$ & $\begin{array}{c}0.8956 \\
(0.8164)\end{array}$ & $\begin{array}{c}-0.1097 \\
(-0.0493)\end{array}$ \\
\hline & & & $\begin{array}{c}0.8965 \\
(0.9133)\end{array}$ & $\begin{array}{c}-0.0927 \\
(-0.2723)\end{array}$ & $\begin{array}{c}0.9120 \\
(1.0593)\end{array}$ & $\begin{array}{c}-0.1953 \\
(-0.5050)\end{array}$ & $\begin{array}{c}0.8941 \\
(0.9000)\end{array}$ \\
\hline & & & $\begin{array}{c}-0.0100 \\
(-0.0093)\end{array}$ & $\begin{array}{c}1.1509 \\
(0.9073)\end{array}$ & $\begin{array}{c}-0.6389 \\
(-0.5050)\end{array}$ & $\begin{array}{c}2.2860 \\
(1.8900)\end{array}$ & $\begin{array}{c}-0.7249 \\
(-0.5181)\end{array}$ \\
\hline & & & $\begin{array}{c}0.0520 \\
(0.0462)\end{array}$ & $\begin{array}{c}-0.0259 \\
(0.0258)\end{array}$ & $\begin{array}{c}0.9883 \\
(0.9000)\end{array}$ & $\begin{array}{c}-0.7249 \\
(-0.5181)\end{array}$ & $\begin{array}{c}2.1987 \\
(1.9489)\end{array}$ \\
\hline
\end{tabular}

stochastic processes with continuous spectrum were obtained as special cases of our general mixed spectrum results, and were obtained using tools notably different than those used by others.

As noted at the beginning of this paper, mixed processes are commonplace in application areas ranging from machinery condition monitoring and communication systems, to climate models and biomedical signal processing, to name a few. In all of these areas spectral information has long played a key role. In some areas (e.g., military specifications for machinery noise and vibration levels) both sample mean and variance are used routinely. AR spectra have been used as high-resolution alternatives to averaged periodogram estimate for the last three decades. For both types of spectral estimation, the mixed process setting poses some complications which have been largely ignored. This is especially true of related spectral statistics. At frequencies sufficiently removed from sinusoid frequencies the results of [1] and [3] can be used. But there is the issue of what is meant by sufficient. This work provides the tools to answer this question in a quantitative manner. The use of spectral families, specifically the AR and MV families, as demonstrated in [14]-[16], represents a relatively new and as yet not popular approach to characterizing the spectral information associated with a mixed process. The fact that the results in this work apply to a wide range of model orders allows one to use it to develop statistically robust sinusoid detection methods for the case of arbitrary noise of unknown color, such as was done in [17]. Such methods could be notably improved if one could obtain the joint spectral statistics of family members, as well as the behavior of the mean and variance of the family as $N, p \rightarrow \infty$. Partial results along these lines are contained in [18].

\section{APPENDIX}

Let $(\cdot)^{\prime}$ denote transpose, $(\cdot)^{*}$ denote complex conjugate.

Proof of Theorem 2: First note that since $\sqrt{N}\left(\hat{\boldsymbol{r}}_{p}^{y}-\boldsymbol{r}_{p}^{y}\right)$ converges in distribution, as stated by Theorem 1, it then fol- lows [19] that $\hat{\boldsymbol{r}}_{p}^{y}=\boldsymbol{r}_{p}^{y}+O_{p}(1 / \sqrt{N})$. Define the following quantities:

$$
\begin{aligned}
g\left(\hat{\boldsymbol{r}}_{p}^{y}\right)= & -\hat{\boldsymbol{R}}_{y}^{-1} \hat{\boldsymbol{r}}^{y}=\hat{\boldsymbol{a}}_{p} \\
g\left(\boldsymbol{r}_{p}^{y}\right)= & -\boldsymbol{R}_{y}^{-1} \boldsymbol{r}^{y}=\boldsymbol{a}_{p} \\
\boldsymbol{\Delta}_{p, i}= & (p \times p) \text { matrix with } \pm i \text { th off-diagonal elements } \\
& \text { equal to } 1, \text { and } 0 \text { otherwise } \\
\boldsymbol{k}_{p, i}= & (p \times 1) \text { vector with } i \text { th element } \\
& \text { equal to } 1, \text { and } 0 \text { otherwise. }
\end{aligned}
$$

Note in particular that $\boldsymbol{\Delta}_{p, 0}$ is the identity matrix, $\boldsymbol{\Delta}_{p, p}$ is a zero matrix, and $\boldsymbol{k}_{p, 0}$ is a zero vector. Then we have [20] for $i=0,1, \ldots, p$

$$
\begin{aligned}
\frac{\partial \hat{\boldsymbol{R}}_{y}^{-1}}{\partial \hat{r}_{i}^{y}} & =-\hat{\boldsymbol{R}}_{y}^{-1} \boldsymbol{\Delta}_{p, i} \hat{\boldsymbol{R}}_{y}^{-1} \\
\frac{\partial \hat{\boldsymbol{r}}^{y}}{\partial \hat{r}_{i}^{y}} & =\boldsymbol{k}_{p, i} .
\end{aligned}
$$

Thus, by the chain rule

$$
\begin{aligned}
\left.\frac{\partial g\left(\hat{\boldsymbol{r}}_{p}^{y}\right)}{\partial \hat{\boldsymbol{r}}_{i}^{y}}\right|_{\hat{\boldsymbol{r}}_{p}^{y}=r_{p}^{y}} & =\left.\left\{\left[-\frac{\partial \hat{\boldsymbol{R}}_{y}^{-1}}{\partial \hat{r}_{i}^{y}}\right] \hat{\boldsymbol{r}}^{y}-\hat{\boldsymbol{R}}_{y}^{-1}\left[\frac{\partial \hat{\boldsymbol{r}}^{y}}{\partial \hat{r}_{i}^{y}}\right]\right\}\right|_{\hat{r}_{p}^{y}=r_{p}^{y}} \\
& =\boldsymbol{R}_{y}^{-1} \boldsymbol{\Delta}_{p, i} \boldsymbol{R}_{y}^{-1} \boldsymbol{r}^{y}-\boldsymbol{R}_{y}^{-1} \boldsymbol{k}_{p, i} \\
& =-\boldsymbol{R}_{y}^{-1}\left(\boldsymbol{\Delta}_{p, i} \boldsymbol{a}_{p}+\boldsymbol{k}_{p, i}\right)
\end{aligned}
$$

Applying [1, Proposition 6.1.6] we then have

$$
\begin{aligned}
\sqrt{N} & \left(\hat{\boldsymbol{a}}_{p}-\boldsymbol{a}_{p}\right) \\
= & -\sum_{i=0}^{p} \boldsymbol{R}_{y}^{-1}\left(\Delta_{p, i} \boldsymbol{a}_{p}+\boldsymbol{k}_{p, i}\right) \sqrt{N}\left(\hat{r}_{i}^{y}-r_{i}^{y}\right)+o_{p}(1) \\
= & -\boldsymbol{R}_{y}^{-1}\left[\boldsymbol{a}_{p}\left(\boldsymbol{\Delta}_{p, 1} \boldsymbol{a}_{p}+\boldsymbol{k}_{p, 1}\right) \cdots\left(\boldsymbol{\Delta}_{p, p} \boldsymbol{a}_{p}+\boldsymbol{k}_{p, p}\right)\right] \\
& \cdot \sqrt{N}\left(\hat{\boldsymbol{r}}_{p}^{y}-\boldsymbol{r}_{p}^{y}\right)+o_{p}(1) .
\end{aligned}
$$



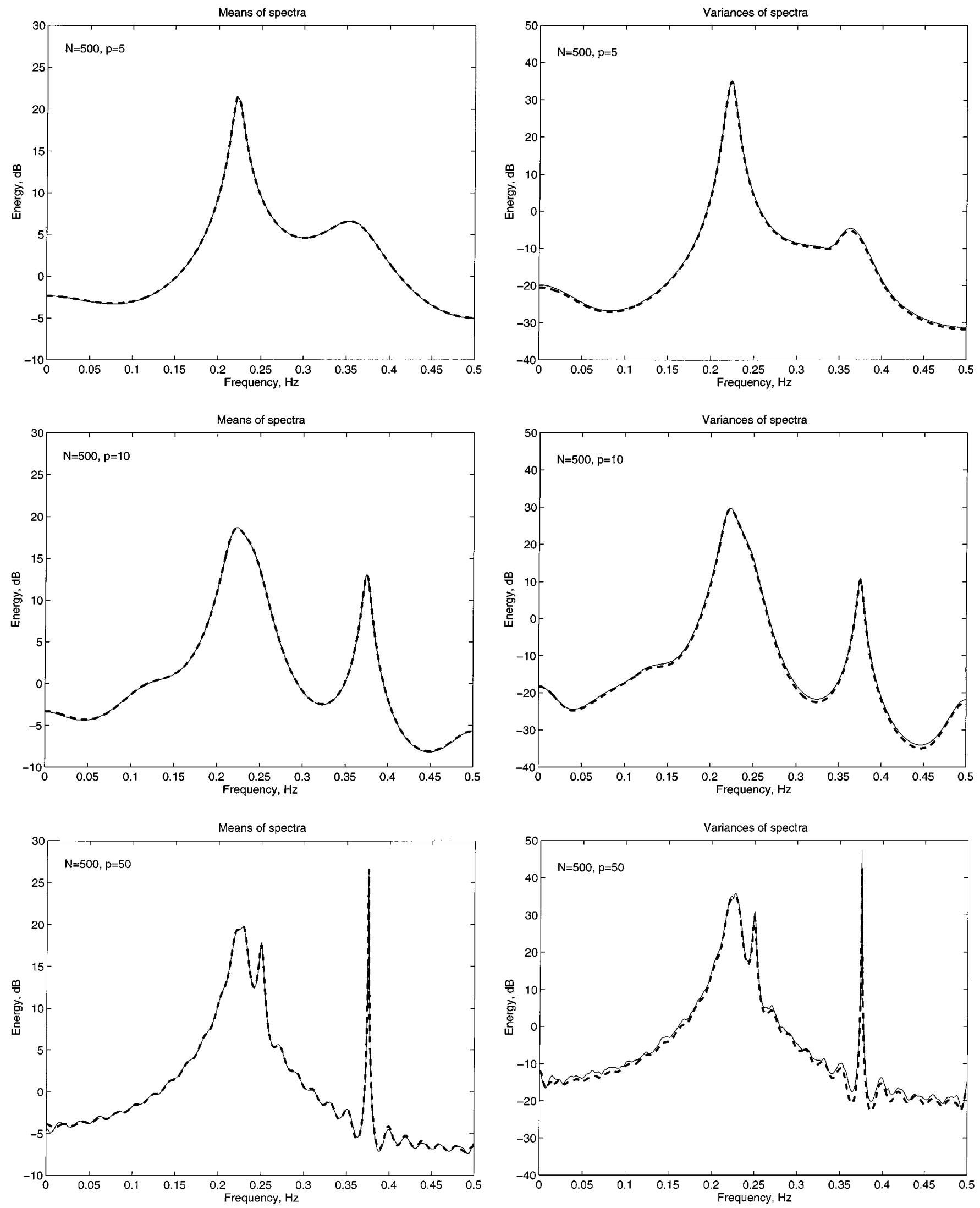

Fig. 2. Case 1: Theoretical (dashed) and estimated (solid) spectra's means and variances of $\operatorname{AR}(p)$ model; Signal frequencies $=0.25 \mathrm{~Hz}, 0.375 \mathrm{~Hz}$; SNR $=$ $-7.3221 \mathrm{~dB} ; N=500$. 
Letting $a_{i}=0$ for $i<0$ and $i>p$, the result follows by using Theorem 1 and by observing that

$$
\boldsymbol{\Delta}_{p, i} \boldsymbol{a}_{p}+\boldsymbol{k}_{p, i}=\left[\begin{array}{c}
a_{1+i} \\
a_{2+i} \\
\vdots \\
a_{p+i}
\end{array}\right]+\left[\begin{array}{c}
a_{1-i} \\
a_{2-i} \\
\vdots \\
a_{p-i}
\end{array}\right], \quad \text { for } i=1, \ldots, p .
$$

Proof of Lemma 1: The proof of this result is similar to that of Theorem 2. First define the function $f$ such that

$$
\begin{aligned}
f\left(\hat{\boldsymbol{r}}_{p}^{y}\right) & =\hat{\boldsymbol{r}}^{y^{\prime}} \hat{\boldsymbol{a}}_{p}=-\hat{\boldsymbol{r}}^{y^{\prime}} \hat{\boldsymbol{R}}_{y}^{-1} \hat{\boldsymbol{r}}^{y} \\
f\left(\boldsymbol{r}_{p}^{y}\right) & =\boldsymbol{r}^{y^{\prime}} \boldsymbol{a}_{p}=-\boldsymbol{r}^{y^{\prime}} \boldsymbol{R}_{y}^{-1} \boldsymbol{r}^{y} .
\end{aligned}
$$

Then, using the chain rule and (14), we have the following partial derivative:

$$
\begin{aligned}
& \left.\frac{\partial f\left(\hat{\boldsymbol{r}}_{p}^{y}\right)}{\partial \hat{r}_{i}^{y}}\right|_{\hat{\boldsymbol{r}}_{p}^{y}=r_{p}^{y}} \\
& \quad=\left.\left\{-\left[\frac{\partial \hat{\boldsymbol{r}}^{y^{\prime}}}{\partial \hat{r}_{i}^{y}}\right] \hat{\boldsymbol{R}}_{y}^{-1} \hat{\boldsymbol{r}}^{y}+\hat{\boldsymbol{r}}^{y^{\prime}}\left[\frac{\partial\left(-\hat{\boldsymbol{R}}_{y} \hat{\boldsymbol{r}}^{y}\right)}{\partial \hat{r}_{i}^{y}}\right]\right\}\right|_{\hat{r}_{p}^{y}=r_{p}^{y}} \\
& =\boldsymbol{k}_{p, i}^{\prime} \boldsymbol{a}_{p}-\boldsymbol{r}^{y^{\prime}} \boldsymbol{R}_{y}^{-1}\left(\boldsymbol{\Delta}_{p, i} \boldsymbol{a}_{p}+\boldsymbol{k}_{p, i}\right) \\
& =2 \boldsymbol{k}_{p, i}^{\prime} \boldsymbol{a}_{p}+\boldsymbol{a}_{p}^{\prime} \boldsymbol{\Delta}_{p, i} \boldsymbol{a}_{p} .
\end{aligned}
$$

Using [1, Proposition 6.1.6], we have

$$
\begin{aligned}
\sqrt{N} & \left(\hat{\boldsymbol{r}}^{y^{\prime}} \hat{\boldsymbol{a}}_{p}-\boldsymbol{r}^{y^{\prime}} \boldsymbol{a}_{p}\right) \\
= & \sum_{i=0}^{p}\left(2 \boldsymbol{k}_{p, i}^{\prime} \boldsymbol{a}_{p}+\boldsymbol{a}_{p}^{\prime} \boldsymbol{\Delta}_{p, i} \boldsymbol{a}_{p}\right) \sqrt{N}\left(\hat{r}_{i}^{y}-r_{i}^{y}\right)+o_{p}(1) \\
= & \left(\sum_{j=1}^{p} a_{j}^{2}\right) \sqrt{N}\left(\hat{r}_{0}^{y}-r_{0}^{y}\right) \\
& +\sum_{i=1}^{p-1} 2\left(a_{i}+\sum_{j=1}^{p-i} a_{j} a_{j+i}\right) \sqrt{N}\left(\hat{r}_{i}^{y}-r_{i}^{y}\right) \\
& +2 a_{p} \sqrt{N}\left(\hat{r}_{p}^{y}-r_{p}^{y}\right)+o_{p}(1) \\
= & \left(g_{0}^{a}-1\right) \sqrt{N}\left(\hat{r}_{0}^{y}-r_{0}^{y}\right) \\
& +\sum_{i=1}^{p} 2 g_{i}^{a} \sqrt{N}\left(\hat{r}_{i}^{y}-r_{i}^{y}\right)+o_{p}(1)
\end{aligned}
$$

where

$$
g_{i}^{a}=\sum_{j=0}^{p-i} a_{j} a_{j+i}=\sum_{j=\infty}^{\infty} a_{j} a_{j+i}
$$

with $a_{0} \equiv 1, a_{i}=0$ for $i<0$ and $i>p$.
Proof of Theorem 3: Using (1b), (2b) and Lemma 1, we have

$$
\begin{aligned}
\sqrt{N}\left(\hat{\sigma}_{p}^{2}-\sigma_{p}^{2}\right) & =\sqrt{N}\left[\left(\hat{\boldsymbol{r}}_{0}^{y}-r_{0}^{y}\right)+\left(\hat{\boldsymbol{r}}^{y^{\prime}} \hat{\boldsymbol{a}}_{p}-\boldsymbol{r}^{y^{\prime}} \boldsymbol{a}_{p}\right)\right] \\
& =\sqrt{N}\left(\hat{\boldsymbol{r}}_{0}^{y}-r_{0}^{y}\right)+\boldsymbol{\beta} \sqrt{N}\left(\hat{\boldsymbol{r}}_{p}^{y}-\boldsymbol{r}_{p}^{y}\right)+o_{p}(1) \\
& =\nu^{\prime} \sqrt{N}\left(\hat{\boldsymbol{r}}_{p}^{y}-\boldsymbol{r}_{p}^{y}\right)+o_{p}(1)
\end{aligned}
$$

and the limiting distribution with the time-domain expression for $\Psi_{\hat{\sigma}_{p}^{2}}$ follows from Theorem 1 .

Proof of Corollary 1: The frequency-domain expression for $\Psi_{\hat{\sigma}_{p}^{2}}$ follows immediately as a consequences of Remark 1 and the frequency-domain expression (8) for $\boldsymbol{\Sigma}$.

Proof of Lemma 2: First recall from Remark 1 that

$$
\left|\rho_{p}(\omega)\right|^{2}=\left[g_{0}^{a} 2 g_{1}^{a} \cdots 2 g_{p}^{a}\right] \gamma(\omega)
$$

where $g_{i}^{a}=\sum_{j=-\infty}^{\infty} a_{j} a_{j+i}$ with $a_{0} \equiv 1, a_{i}=0$ for $i<0$ and $i>p$. Using [1, Proposition 6.1.6], we now have

$$
\begin{aligned}
\sqrt{N}\left[\left|\hat{\rho}_{p}(\omega)\right|^{2}-\left|\rho_{p}(\omega)\right|^{2}\right]=\sum_{k=1}^{p} \frac{\partial}{\partial a_{k}} \\
\cdot\left\{\left[g_{0}^{a} 2 g_{1}^{a} \cdots 2 g_{p}^{a}\right] \gamma(\omega)\right\} \sqrt{N}\left(\hat{a}_{k}-a_{k}\right)+o_{p}(1) .
\end{aligned}
$$

Now, since $\partial g_{i}^{a} / \partial a_{k}=a_{k+i}+a_{k-i}$ we then have

$$
\begin{aligned}
\frac{\partial}{\partial a_{k}}\left\{\left[g_{0}^{a} 2 g_{1}^{a}\right.\right. & \left.\left.\cdots 2 g_{p}^{a}\right] \boldsymbol{\gamma}(\omega)\right\} \\
& =2\left[a_{k}\left(a_{k+1}+a_{k-1}\right) \cdots\left(a_{k+p}+a_{k-p}\right)\right] \boldsymbol{\gamma}(\omega)
\end{aligned}
$$

where the row vector of $a_{i}^{\prime} s$ is just the $k$ th row of the matrix $A$. Then, (10) follows by a rearrangement in matrix notation. Finally, (11) follows from some basic asymptotic results of [1, Ch. 6].

Proof of Theorem 4: First note from (5) and Lemma 2 that

$$
\begin{aligned}
\sqrt{N}\left[\left|\hat{\rho}_{p}(\omega)\right|^{2}-\right. & \left.\left|\rho_{p}(\omega)\right|^{2}\right] \\
& =-2[\boldsymbol{A} \gamma(\omega)]^{\prime} \boldsymbol{R}_{y}^{-1} \boldsymbol{A} \sqrt{N}\left(\hat{\boldsymbol{r}}_{p}^{y}-\boldsymbol{r}_{p}^{y}\right)+o_{p}(1) .
\end{aligned}
$$

Then, together with (15), we have

$$
\begin{aligned}
\sqrt{N}\left[\begin{array}{c}
\left(\hat{\sigma}_{p}^{2}-\sigma_{p}^{2}\right) \\
\left(\left|\hat{\rho}_{p}(\omega)\right|^{2}-\left|\rho_{p}(\omega)\right|^{2}\right)
\end{array}\right] & \stackrel{d}{\longrightarrow} \mathrm{N}\left(\mathbf{0},\left[\begin{array}{cc}
\Psi_{\hat{\sigma}_{p}^{2}} & \Psi_{\hat{\sigma}_{p}^{2},\left|\hat{\rho}_{p}\right|^{2}} \\
\Psi_{\hat{\sigma}_{p}^{2},\left|\hat{\rho}_{p}\right|^{2}} & \Psi_{\left|\hat{\rho}_{p}\right|^{2}}
\end{array}\right]\right) .
\end{aligned}
$$

Now, using yet again [1, Proposition 6.1.6], we obtain our final result from

$$
\begin{aligned}
& \sqrt{N}\left[\hat{S}_{p}(\omega)-S_{p}(\omega)\right]=\frac{1}{\sigma_{p}^{2}} S_{p}(\omega)\left[1-S_{p}(\omega)\right] \\
& \cdot \sqrt{N}\left[\begin{array}{c}
\left(\hat{\sigma}_{p}^{2}-\sigma_{p}^{2}\right) \\
\left(\left|\hat{\rho}_{p}(\omega)\right|^{2}-\left|\rho_{p}(\omega)\right|^{2}\right)
\end{array}\right]+o_{p}(1) .
\end{aligned}
$$




\section{REFERENCES}

[1] P. J. Brockwell and R. A. Davis, Time Series: Theory and Methods, 2nd ed. New York: Springer-Verlag, 1991.

[2] P. E. Caines, Linear Stochastic Systems. New York: Wiley, 1988.

[3] D. B. Percival and A. T. Walden, Spectral Analysis for Physical Applications: Multi-Taper and Conventional Univariate Techniques. New York: Cambridge Univ. Press, 1993.

[4] A. B. Baggeroer, "Confident interval for regression (MEM) spectral estimates," IEEE Trans. Inform. Theory, vol. IT-22, pp. 534-545, Sept. 1976.

[5] K. N. Berk, "Consistent autoregressive spectral estimates," Ann. Statist., vol. 2, pp. 489-502, 1974.

[6] H. Sakai, "Statistical properties of AR spectral analysis," IEEE Trans. Acoust., Speech, Signal Processing, vol. ASSP-27, pp. 402-409, 1979.

[7] M. Mackisack and D. S. Poskitt, "Some properties of autoregressive estimates for processes with mixed spectra," J. Time Ser. Anal., vol. 11, pp. 325-337, 1990

8] C. Foias, A. E. Frazho, and P. J. Sherman, "A geometric approach to the maximum likelihood spectral estimator for sinusoids in noise," IEEE Trans. Inform. Theory, vol. 34, pp. 1066-1070, Sept. 1988.

[9] P. J. Sherman, L. White, J. Spanjaard, and R. Bitmead, "Asymptotic statistics of AR spectral estimators for processes containing mixed spectrum," in Proc. 8th IEEE Signal Processing Workshop on Statistical Array Processing, Corfu, Greece, 1996, pp. 48-51.

[10] T. H. Li, B. Kedem, and S. Yakowitz, "Asymptotic normality of sample autocovariances with an application in frequency estimation," Stoch. Processes Applic., vol. 52, pp. 329-349, 1994.
[11] T. H. Li, "Bartlett-type formulas for complex multivariate time series of mixed spectra," Statist. Probab. Lett., pp. 259-268, 1996.

[12] P. J. Sherman, "Circulant approximations of the inverses of Toeplitz matrices and related quantities with applications to stationary random processes," IEEE Trans. Acoust., Speech, Signal Processing, vol. ASSP-33, pp. 1630-1632, Dec. 1985.

[13] P. J. Sherman and K. L. Lou, "On the family of ML spectral estimates for mixed spectrum identification," IEEE Trans. Acoust., Speech, Signal Processing, vol. 39, pp. 644-655, 1991.

[14] P. J. Sherman and L. B. White, "Periodic spectral analysis of diesel vibration data," J. Acoust. Soc. Amer., vol. 98, no. 6, pp. 3285-3301, Dec. 1995.

[15] C. Wikle and P. J. Sherman, "Using the family of MV spectra to determine periodicities in atmospheric data," J. Climate, vol. 8, no. 10, pp. 2352-2363, Oct. 1995

[16] D. Van Zante, R. Feddersen, M. Suarez, and P. Sherman, "The stochastic structure of downstream pressure from an axial compressor: Part I," Mech. Syst. Signal Processing, vol. 10, no. 4, pp. 413-422, July 1996.

[17] L. White and P. Sherman, "Detection of sinusoids in unknown colored noise using ratios of AR spectrum estimates," in Proc. 1999 Conf. Information, Decision and Control, Adelaide, Australia, Feb. 8-10, 1999.

[18] X. Liu and P. Sherman, "Asymptotic statistical properties of the Capon MV spectral estimator for mixed random processes," in Proc. 9th IEEE Signal Processing Workshop on Statistical Signal and Array Processing, Portland, OR, Sept. 14-16, 1998, pp. 328-331.

[19] R. J. Serfling, Approximation Theorems of Mathematical Statistics. New York: Wiley, 1980

[20] F. A. Graybill, Matrices With Applications in Statistics, 2nd ed. Belmont, CA: Wadsworth, 1983. 